To appear in proceedings of CSMR 2003

\title{
Using A Taxonomy Tool To Identify Changes in OO Software
}

\author{
Peter Clarke, Brian Malloy \\ Computer Science Department \\ Clemson University \\ Clemson, SC 29634 \\ U.S.A. \\ $\{$ peterc, malloy\}@cs.clemson.edu
}

\author{
Paul Gibson \\ Computer Science Department \\ National University of Ireland \\ Maynooth, Co. Kildare \\ Ireland \\ pgibson@cs.may.ie
}

\begin{abstract}
In this paper, we present a taxonomy that allows the maintainer to catalog $O O$ classes based on the characteristics of the class. The characteristics of a class include the properties of data items and methods, as well as the relationships with other classes in the application. We construct a tool to track changes across multiple releases of software applications containing hundreds of classes, providing information about each changed class. Our tool identifies class changes in terms of the characteristics exhibited by classes with the same name in different releases of an application.
\end{abstract}

\section{Introduction}

It is widely acknowledged that software maintenance occupies a large fraction of the software development cycle $[2,20]$, where maintenance includes modifying, extending, debugging, testing, and documenting the application. Wilde et al. identify several factors that affect the maintenance effort in OO software; these include: high-level system understanding, locating system functionality, and detailed code understanding [20]. In addition to the difficulties associated with code comprehension in pre-OO software, the OO paradigm has presented new challenges such as understanding class hierarchies, polymorphism, and other complex dependencies between entities in an OO program [20].

Several class abstraction methods are used during the maintenance process to assist in understanding the source code of an application. These include graphical languages, such as the Unified Modeling Language, or UML [16] and OO design metrics (OODMs) [5]. UML contains a plethora of graphs and diagrams for visualizing, specifying, and designing artifacts of a software intensive system, including the class diagram used to represent relationships between OO classes. OODMs generated from software applications are used mainly to determine or measure the quality of a software application [5].

The problem with class diagrams is that for applications with more than twenty classes the diagrams become cluttered, difficult to interpret and ineffective [11]. The typical maintenance activity includes hundreds or even thousands of classes making class diagrams ineffective. Comparing OODMs for two applications identifies changes at a more abstract level than class diagrams, for example identifying the number of public methods added to a class. OODMs are more suited to providing information on qualities of software, such as, reusability, maintainability and testability [5].

In this paper, we provide an alternative representation to facilitate software maintenance. We present a taxonomy that allows the maintainer to catalog OO classes based on the characteristics of the class. The characteristics of a class include the properties of data items and methods, as well as the relationships with other classes. Using our taxonomy tool we track changes across multiple releases of applications containing hundreds of classes.

Unlike previous approaches that capture information about the control structure of methods in a class $[9,15]$, our taxonomy allows us to construct a summary of the characteristics of the class. Our approach also differs from the information provided by tools such as JavaDocs [1] and WinCV (for MS.Net) [14] since we perform analysis on the code 
while summarizing the characteristics of the class. For example, we flatten inheritance hierarchies to accurately capture the characteristics visible in a derived class.

In the next section, we identify class characteristics, introduce class changes during maintenance and define the term taxonomy. In Section 3 we present our taxonomy and in Section 4 overview our taxonomy tool providing a simple example. In Section 5 we describe the results of our case study. In the penultimate section we compare our work to previous research and in the last section state some concluding remarks.

\section{Background}

The widespread use of the OO paradigm to develop software has resulted in new challenges during software maintenance. One such challenge is tracking changes to classes during the maintenance of OO software. In this section we identify the characteristics of a class, briefly describe how changes are used during maintenance, and introduce the concept of a taxonomy.

\subsection{Class Characteristics}

Meyer defines a class as a static entity that represents an abstract data type with a partial or total implementation [12]. The static description supplied by a class should include a specification of the features that each object will contain. These features fall into two categories: (1) attributes, and (2) routines. Attributes are referred to as data items and instance variables in other $\mathrm{OO}$ languages while routines are referred to as member functions and methods. Throughout this paper we will use the terms attributes and routines.

We define the characteristics for a given class $C$ as the properties of the features in $C$ and the relationships $C$ has with other classes in the implementation. The properties of the features in $C$ describe how criteria such as types, accessibility, shared class data, deferred features, dynamic binding, polymorphism, exception handling, and concurrency are represented in the attributes and routines of $C[12]$. The relationships of $C$ with other classes include associations, dependencies, and generalizations. We define these relationships based on the definitions given by Rumbaugh et al. [16].

\subsection{Class Changes During Maintenance}

Recent research in the area of software maintenance has focussed on the problems of change impact analysis [9, 10] and regression testing [6, 15]. Both of these areas of research deal with identifying changes made to existing software. Change impact analysis (CIA) focuses on how a change in the implementation will affect the semantics of the software system [17]. The results from CIA can be valuable in predicting the risk and cost associated with the proposed software changes [10]. Most of the current research in regression testing deals with selective retest techniques [15]. The objective of selective retest techniques is to reduce the cost of regression testing by reusing appropriate test cases to test the modified code [15].

Identifying changes in OO software systems is challenging because of the complex dependencies that exist between program entities. Change identification for classes is further compounded by the class characteristics mentioned in the previous section. Researchers have developed novel ways to represent and report these changes at various levels of granularity $[9,15]$. One approach not yet fully exploited in the literature is the identification of changes based on a taxonomy of OO classes.

\subsection{Taxonomy}

A taxonomy is a scientific method of classification according to an established system in a specific domain, with the resulting catalog used to provide a framework for analysis. Any taxonomy should take into account the importance of separating elements of a group (taxon) into subgroups (taxa) that are mutually exclusive, unambiguous, and taken together include all possibilities[19].

\section{Taxonomy of OO Classes}

In this section we describe a taxonomy that allows the maintainer to catalog a class, written in virtually any OO language, based on the characteristics of that class. Using the cataloged entries for the same class in different versions of a software application the maintainer can identify changes based on the characteristics of the class (see Section 2.1). 
The taxonomy presented in this paper is an extension to the one in [3].

Each class cataloged using our taxonomy consists of three components: (1) Class - identifies the fully qualified name of the class, (2) Nomenclature identifies the group (or taxon) the class belongs to, and (3)Feature Properties - a list of sub-groups categorizing the attributes and routines of the class. We use a string of descriptors in the Nomenclature and Feature Properties components to describe the characteristics exhibited by a given class. To describe a class written in virtually any OO language the descriptors are divided into two groups: (1) core - identifies characteristics found in most OO languages, and (2)add-ons - descriptors specific to a language.

\subsection{Nomenclature}

The Nomenclature of a class identifies the group (or taxon) the class belongs to, as well as provides a summary of class properties and relationships with other classes in the application. The Nomenclature consists of two parts: (1)Modifier - summarizes class properties and relationships the class has with other classes, (2) Type Family - identifies the types associated with the class. The following core descriptors are used in the Modifier part of the Nomenclature:

- Generic - identifies a class that takes formal generic parameters for arbitrary types.

- Concurrent-identifies a class whose instances are threads/processes.

- Inheritance-free - indicates a class is not part of an inheritance hierarchy.

- Parent-identifies a class that is the root class of an inheritance hierarchy.

- External Child - identifies a class that is a descendant of a parent and has no descendant classes.

- Internal Child - identifies a class that is a descendant as well as a parent.

- Abstract - identifies a class that contains deferred features.

Some of the add-on descriptors for the $\mathrm{C}++$ language include Nested, Multi-Parents, Friend, and Has_Friend.
The Type Family part of the Nomenclature represents a summary of the types associated with the class. These types are used in the declarations of attributes and routine locals (parameters and variables). The type families used in the taxonomy are:

- Family NA - no associated types used.

- Family $P$ - scalar primitive types e.g., int.

- Family $P^{*}$ - non-scalar primitive types, including reference types and arrays of primitive types.

- Family $U$ - user-defined types i.e., classes.

- Family $U^{*}$ - references to user-defined types.

- Family L - class libraries, e.g., STL[8].

- Family $L^{*}$ - references to class libraries.

- Family $A$ - any type (used as parameters for generics).

- Family $A^{*}$ - references to any type.

The Type Family also indirectly identifies relationships with other categories of classes including composition, aggregation, and parameterization. For example, if an attribute in a class is a user defined type (i.e., U) then composition exists.

Figure 1 illustrates how the descriptors and type families are combined in the Nomenclature component. In addition, Figure 1 shows how our taxonomy catalogs $\mathrm{OO}$ classes into mutually exclusive groups (or taxa). An example of one such group is Non-Generic Sequential Concrete Inheritance-Free Families $P$, shown along the top branch of the tree in Figure 1. We consider the descriptors Non-Generic, Sequential, and Concrete, as defaults descriptors, hence the Nomenclature becomes InheritanceFree Families $P$. This group represents classes that are not part of an inheritance hierarchy and contain data (attributes and routine locals) whose types are primitive. The descriptors italicized in Figure 1 are considered to be default descriptors and not listed above.

\section{$3.2 \quad$ Feature Properties}

The Feature Properties component of the taxonomy consists of three sections: (1) Attributes a list of subgroups categorizing the attributes, (2) Routines - a list of subgroups categorizing the routines, and (3) Feature Classification - a summary 


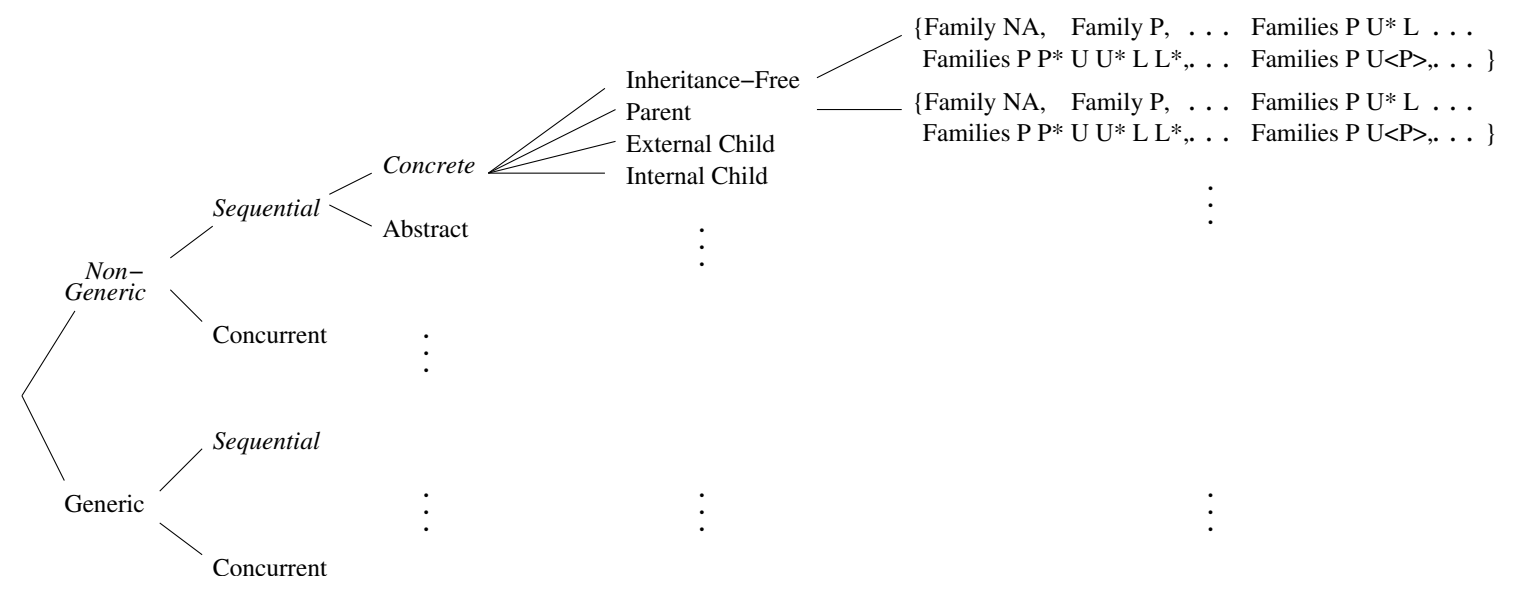

Figure 1. Tree representing structure of Nomenclature for $\mathrm{OO}$ classes. We consider the descriptors in italics as defaults and therefore not stated in the Nomenclature. The type families are enclosed in braces representing all possible combinations, of which one is chosen. Vertical ellipses implies repetition of the tree structure. The type families for Generic classes include families A and A*, representing the unknown parameters in the generic classes

of the inherited features. The properties of the attributes for a class are described using the following descriptors.

- Concurrent - if the object is a thread or process.

- Polymorphic - if the attribute has the potential to be polymorphic. That is, the attribute is a reference to a user defined type $\left(U^{*}\right)$, and the user defined type (class) has children.

- Private, Protected or Public - depending on the accessibility of the attribute.

- Static - if the attribute is shared class data.

- Family NA - represents no class attributes.

- Family P, or Family $P^{*}$, . . . Family $A^{*}$ represents the type family of the attribute.

- Family $\mathrm{m}<\mathrm{n}>$ - represents attributes that are instantiated generic types, where $m$ is type family $\mathrm{U}$ or $\mathrm{L}$, and $\mathrm{n}$ represents any of the type families.

The properties of a routine in the class are described as follows:

- Concurrent - represents a routine that instantiates a thread or process.

- Synchronized - if the routine contains code that is synchronized.
- Exception-R - if the routine contains code that raises an exception.

- Exception-H - if the routine contains code that handles an exception.

- Non-Virtual - identifies a routine that is statically bound.

- Virtual - identifies a routine that is dynamically bound.

- Deferred - if the implementation of the routine is deferred.

- Private, Protected or Public - depending on the accessibility of the routine.

- Static - if the routine is a shared class routine.

- Type family information for parameters and local variables.

We classify the inherited features of a class as outlined in [7]: new - if the feature is declared in the child class, recursive - if the feature is inherited from the parent unchanged, and redefined - if the feature is a routine and has the same signature as the one declared in the parent but with a different implementation. The entries in the Attributes and Routines sections of the taxonomy are classified as either new, recursive or redefined as appropriate. In the Feature Classification section we use None if the class is Inheritance-free and Unknown if the class is inherited from a class in the standard library. 


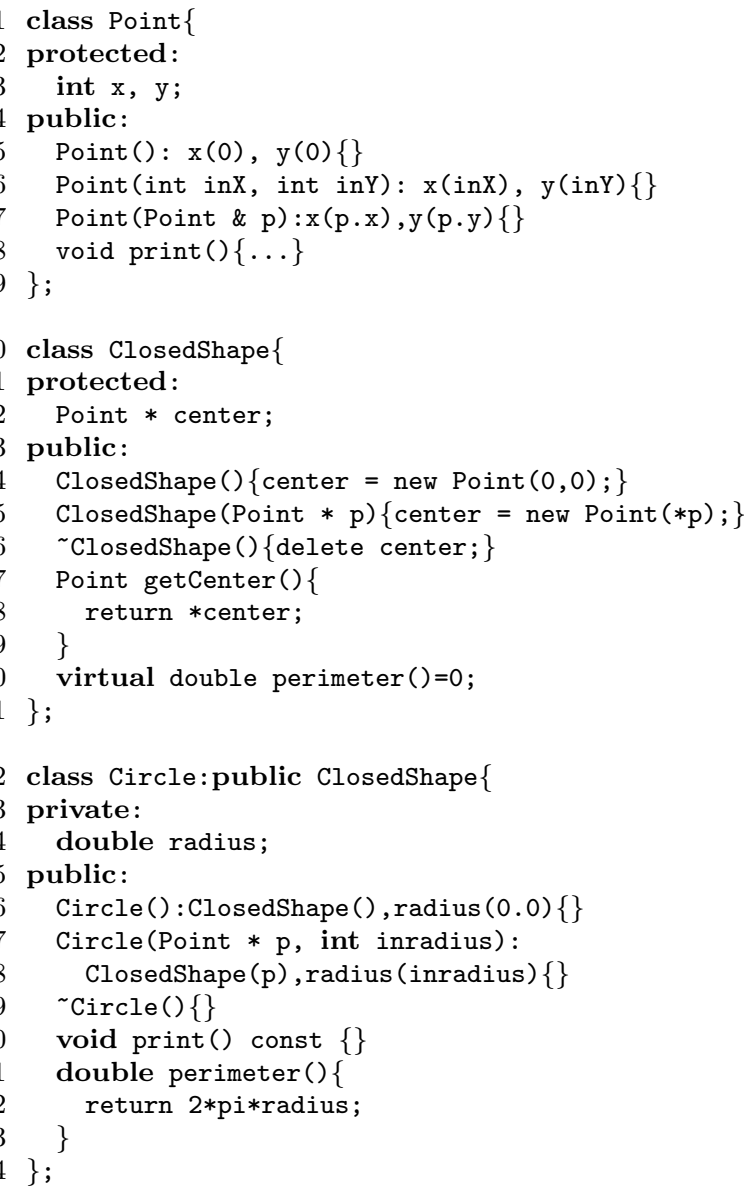

Figure 2. Classes Point, ClosedShape and Circle.

Each entry in the Feature Properties component of the taxonomy is prefixed with a numerical value enclosed in square brackets representing the number of times that category of feature occurred in the class. The add-on descriptors used in the Attributes and Routines sections are enclosed in parentheses, one such add-on for $\mathrm{C}++$ in the Routines section is Constant.

\subsection{An Illustrative Example}

In this section we present an example to illustrate our approach toward cataloging classes using our taxonomy. The example in Figure 2 illustrates $\mathrm{C}++$ code for classes Point, ClosedShape and Circle. Figure 3 illustrates the class Circle cataloged using our taxonomy.
Class: Circle

Nomenclature: External Child Families $P U^{*}$

Feature Properties

Attributes: [1] New Private Family $P$

[1] Recursive Protected Family $U^{*}$

Routines:

[2] New Non-Virtual Public Family NA

[1] New (Constant) Non-Virtual Public Family NA

[1] New Non-Virtual Public Families P $U^{*}$

[1] Recursive Non-Virtual Public Family NA

[1] Redefine Virtual Public Family NA

Feature Classification:

[1] New Attribute

[1] Recursive Attribute

[4] New Non-Virtual Routine

[1] Recursive Non-Virtual Routine

[1] Redefined Virtual Routine

Figure 3. Cataloged entry for class Circle.

In Figure 3, the nomenclature of class Circle is External Child Families $P U^{*}$ since class Circle is inherited from ClosedShape, has no descendents, and the only type families are primitive and pointers to user-defined(see Figure 2) . The Attributes section summarizes those attributes visible in the scope of class Circle; these are radius, a primitive type, and the inherited attribute center a pointer to Point, a user-defined type.

The Routines section captures the information for the routines defined within Circle and those inherited from ClosedShape. For example, Circle() is cataloged as New Non-Virtual Public Family NA and perimeter() as Redefine Virtual Public Family $N A$, while the routine getCenter(), inherited from ClosedShape unchanged, is cataloged as Recursive Non-Virtual Public Family NA. The descriptor Virtual implies the routine is dynamically bound, while Non-Virtual implies static binding. The above entries are Family NA since none of the routines declare any parameters or local variables. The print() routine is cataloged with the property Constant in parentheses since this is a feature peculiar to the language $\mathrm{C}++$. The Feature Classification compo- 
nent identifies the features that are inherited from the class ClosedShape. For example, the routine perimeter() in the class Circle is cataloged as Redefined Virtual Routine since it is declared as pure virtual in ClosedShape and implemented in class Circle. The entry [4] New Non-Virtual Routine refers to the two constructors, destructor, and the print() routine.

\section{Taxonomy Tool}

Our taxonomy tool, referred to as TaxTool, reverse engineers classes of a $\mathrm{C}++$ software application, cataloging them using our taxonomy. The tool also has the ability to compare two versions of a $\mathrm{C}++$ application and identify those entities that have changed with respect to the class characteristics captured by the taxonomy. Figure 4 is a UML class diagram that illustrates the important subsystems of TaxTool.

\subsection{The Clouseau API}

The Clouseau ${ }^{1}$ application programmers interface (API), was designed to facilitate symbol table inspection of $\mathrm{C}++$ programs [11]. Clouseau provides information about the accessibility, visibility, and types of namespaces, classes, functions, and variables for the program under consideration. With Clouseau, the application programmer is completely separated from the complexity of parsing. The Clouseau API forms a facade for the Keystone parser [13], so that users can access its functionality without the burden of dealing with its complexity[4]. Clouseau users are relieved of the burden of parsing the program, since the API exploits the Keystone parser to provide this functionality. Clouseau is implemented as a Unix ${ }^{\mathrm{TM}}$ shared object.

\subsection{Comparison of Classes}

Tax_Cataloger uses the Clouseau API to access the information stored in keystone's symbol table

\footnotetext{
${ }^{1}$ The Clouseau API is named after Inspector Clouseau, a character in the Pink Panther movies, because the API permits users to "inspect" symbol table information.
}

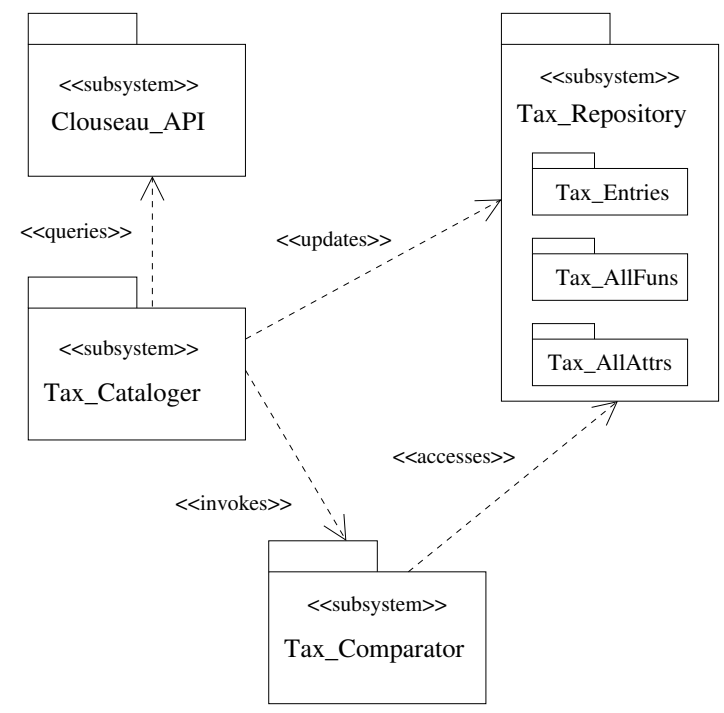

Figure 4. Class diagram for TaxTool.

for each class definition in the $\mathrm{C}++$ applications supplied to the TaxTool. The information provided by Clouseau is used to catalog each class recursively, starting with classes defined in the Global Namespace followed by nested class definitions and finally classes defined in routines. After all the classes have been cataloged Tax_Cataloger then invokes Tax_Comparator to compare the classes in the applications.

Tax_Repository stores an entry in the component Tax_Entries for each class cataloged using the taxonomy. Each entry consists of a Nomenclature and subgroups representing the features of the class. It is essential that we flatten all inheritance hierarchies to accurately catalog inherited features, as a result we store the properties for all attributes and routines in the Tax_AllAttrs and Tax_AllFuns components respectively.

The Tax_Comparator subsystem uses the information stored in Tax_Repository subsystem to compare the applications at two levels of granularity. The information in Tax_Entries allows applications to be compared at the more abstract level, identifying changes in the Nomenclature and Feature Properties components of the taxonomy. Changes identified at this level include relationships between classes e.g., classes becoming part of an inheritance hierarchy, or classes having associations with new type families. Other changes at this level include new or deleted subgroups for attributes and routines. At the finer level of granularity changes 


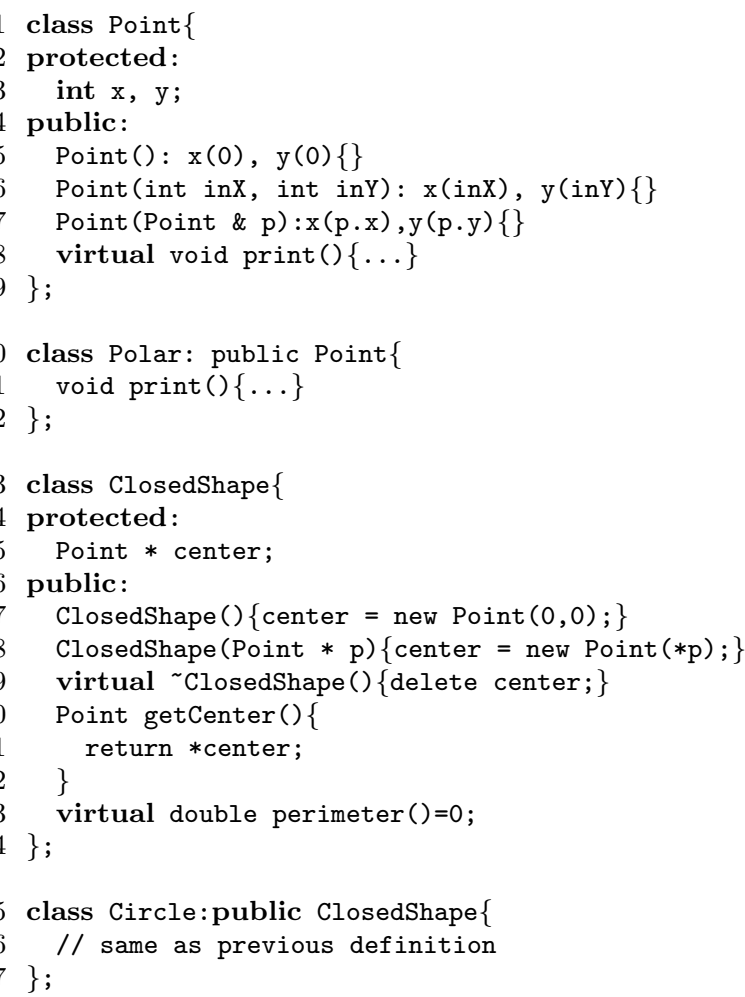

Figure 5. Modified code for classes Point, Polar and ClosedShape.

are identified using the information stored in the Tax_AllAttrs and Tax_AllFuns components. These changes include identifying new or deleted features and changes in the properties of specific features. In the next section we will illustrate examples of the changes TaxTool identifies.

\subsection{Example of Class Changes}

Figure 5 illustrates a modified version of the code shown in Figure 2. The textual changes to the code in Figure 2 include: the conversion of the function print() in class Point from non-virtual to virtual (line 8 of Figures 2 and 5), the conversion of the destructor in class ClosedShape from nonvirtual to virtual (line 16 of Figure 2 and line 18 of Figure 5), and the addition of the class Polar (line 10 of Figure 5) derived from class Point.

Figure 6 is the output generated by TaxTool identifying the changes to the code based on our

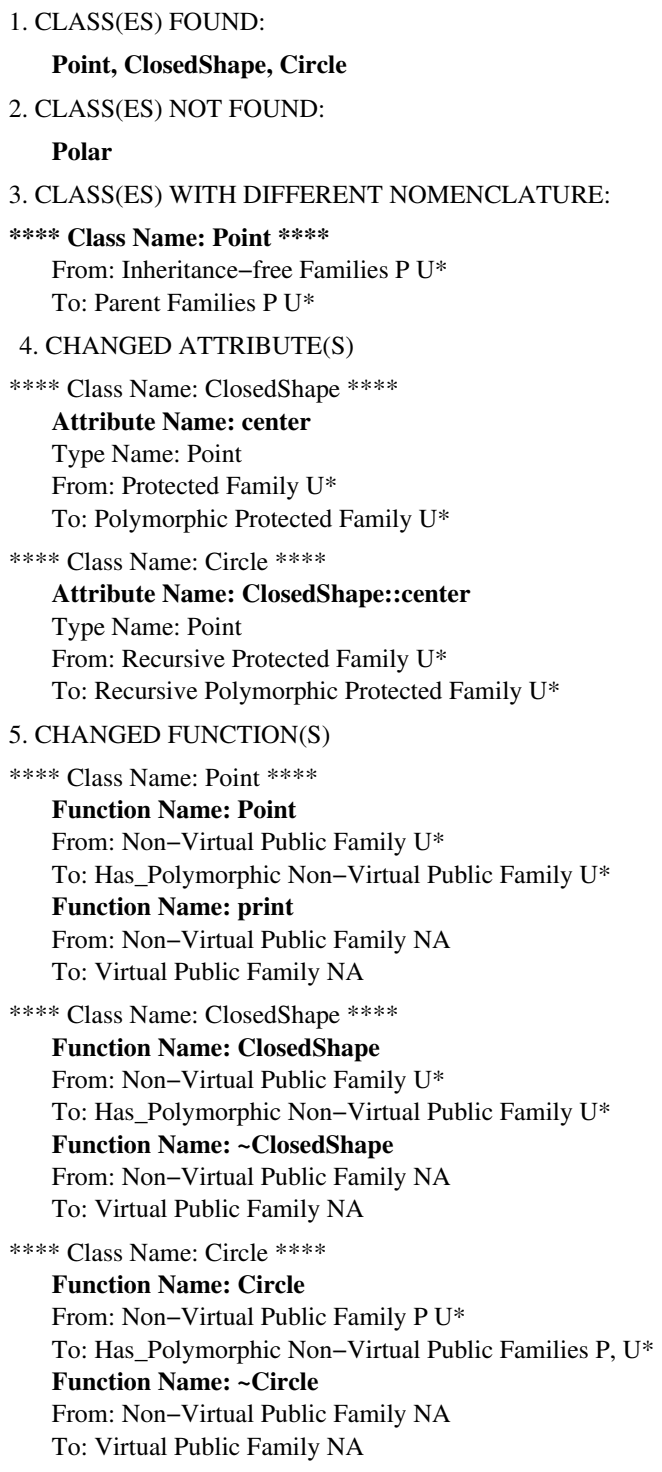

Figure 6. Changes for classes Point, ClosedShape and Circle.

taxonomy and is divided into 5 partitions. Partition 1 of Figure 6 identifies the classes found in both versions of the program and Partition 2 the new classes. Partition 3 lists those classes common to both versions of the program but with a different nomenclature. Class Point has changed from Inheritance-free Families $P U^{*}$ to Parent Families $P U^{*}$, as a result of the new class Polar being derived from Point. The addition of class Polar also affects the attributes in class ClosedShape and Circle, 


\begin{tabular}{||c||c|c|c|c|c|c||}
\hline $\begin{array}{c}\text { Test } \\
\text { Case No. }\end{array}$ & Application & $\begin{array}{c}\text { Library } \\
\text { Release }\end{array}$ & $\begin{array}{c}\text { Release } \\
\text { Date }\end{array}$ & No. Lines & No. Classes & $\begin{array}{c}\text { Classes with } \\
\text { Routines }\end{array}$ \\
\hline 1 & graphdraw & IV Tools 0.7 & Dec. 1998 & 3575 & 156 & 63 \\
2 & graphdraw & IV Tools 1.0.0 & Nov. 2001 & 4356 & 170 & 65 \\
3 & graphdraw & IV Tools 1.0.1 & Jan. 2002 & 4354 & 170 & 65 \\
\hline
\end{tabular}

Table 1. Summary of the test cases used in the case study

see Partition 4 of Figure 6 . The attribute center in ClosedShape now has the potential to be polymorphic hence the descriptor Polymorphic is added to entry for the Attribute component. The attribute center is inherited in Circle, as a result this change is also inherited.

Partition 5 of Figure 6 identifies changes to the entries in the Routines component of our taxonomy for the two versions of the program. The entry for the one-argument constructor of class Point now includes the descriptor Has_Polymorphic, reflecting that references of type Point are potentially polymorphic. Similar changes are also generated for the one-argument constructor of ClosedShape (line 15 Figure 2), and the two-argument constructor of class Circle (line 27 Figure 2). The remaining changes of Partition 5 in Figure 6 represent functions that have become virtual. These functions include print in class Point, the destructor in ClosedShape, and the destructor in Circle derived from ClosedShape.

\section{A Case Study}

In this section, we describe our application suite and the results obtained when various releases of a library are compared using TaxTool. In the next subsection we overview the application suite and experimental conditions used in the case study. In subsection 5.2 we present a summary of the changes identified by our tool.

\subsection{Software Applications}

Table 1 summarizes our test suite including three releases of the library graphdraw[18], a drawing application that uses $I V$ Tools, a suite of free X Windows drawing editors for PostScript, TeX and web graphics production. We emphasize that the three applications remained constant for the experiments; only the libraries changed across the different releases.

The first column of Table 1 lists the number that we associate with each test case, the second column lists the name of the application that uses the respective libraries and the third column lists the release number of the library. For example, test cases 1 through 3 show the graphdraw application that uses releases $0.7,1.0 .0$ and 1.0.1 of the IV Tools library. The fourth column in the table lists the release date of the library and the fifth column lists the number of lines for each application, with blank lines and comments removed. The final columns in Table 1 list the total number of classes and the number of classes with routines, or functions, for each of the test cases.

The experiments in this section were executed on systems running version 7.1 of Red Hat and Solaris SunOS version 5.8. To provide some insight into the efficiency of our taxonomy tool, we were able to compare the graphdraw application that uses release 1.0.0 and 1.0.1 of the IV Tools library in 19.74 seconds on a Dell Precision 530 workstation, with a Xeon $1.7 \mathrm{GHz}$ processor and $512 \mathrm{MB}$ of RDRAM, running the Red Hat 7.1 operating system.

\subsection{Summary of Changes}

One contribution of our taxonomy is to enable the maintainer of a system to abstract the important characteristics of a class. TaxTool allows us to track the changes in these characteristics across multiple releases of an application or library. The graph in Figure 7 shows a summary of the results in comparing these releases. The $\mathrm{x}$-axis represents the changed entities i.e., Nomenclature, Attributes, Routines and Feature Classification components of the taxonomy, and New or Deleted classes. The $\mathrm{y}$-axis represents the number of changed classes. 


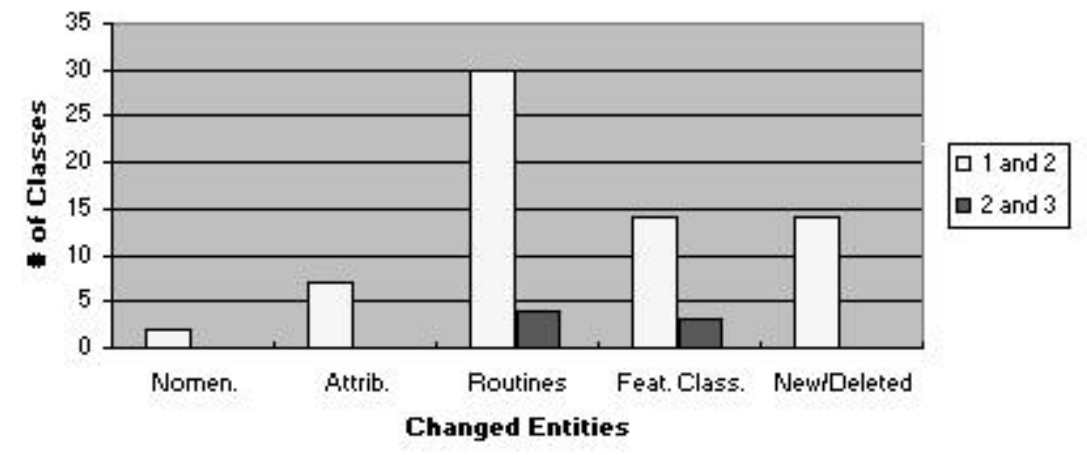

Figure 7. Graphical illustration representing changes in the applications using the various libraries.

Space restrictions limit us from providing details of these changes as illustrated in Figure 6.

To illustrate the impact of the information in Figure 7, consider the leftmost bar of each group representing information about the comparison of test cases 1 and 2. The two changes in the Nomenclature component, represent the following changes: (1) class OverlayKit from Parent Families $P P^{*} U$ $U^{*}$ to (Friend) Parent Families $P P^{*} U U^{*}$, and (2) class OverlayEditor from Internal Child Families $P$ $P^{*} U U^{*}$ to (Has_Friend) Internal Child Families $P P^{*} U U^{*}$. The first change states that OverlayKit in version 1.0.0 of the IV Tools library (test case 2) has become a friend class, while the second change says that OverlayEditor in version 1.0.0 declares another class as a friend. The bar representing changes in class attributes states that seven classes registered changes in the Attributes component. These changes represent 27 attributes being added to the seven classes in version 1.0.0 of the IV Tools library. One such attribute is _clr_button_flag, cataloged as Recursive Protected Family P, added to class GraphKit and inherited from OverlayKit.

The leftmost bar in the group labeled Routines, states that thirty classes registered changes in the
Routines component between releases 0.7 (test case 1) and 1.0.0 (test case 2) of the IV Tools library. A summary of the changes for the routines generated by TaxTool for the thirty classes include: 86 routines whose cataloged entry changed, 12 routines were deleted and 92 routines added. One of the routines that changed was comterp visible in class GraphEditor and inherited from class OverlayEditor. The change was from Recursive (Constant) NonVirtual Public Static Families $P P^{*}$ to Recursive (Constant) Virtual Public Static Families $P P^{*}$. The bar labeled Feat Class for test cases 1 and 2 summarizes the changes captured by comparing the cataloged entries in the Feature Classification components for the classes in test cases 1 and 2 . Fourteen classes registered changes with respect to inherited features. The rightmost bar of Figure 7 indicates the number of new and deleted classes. In this case, fourteen new classes were added to the release 1.0.0 of IV Tools library (test case 2). 


\section{Related Work}

Kung et al. present a technique to track the changes to OO software using a multigraph consisting of an Object Relation Diagram (ORD), Block Branch Diagram (BBD) and Object State Diagram (OSD) [9]. These graphs are used to identify changes in the data, method, class, and class library components of the software. The model can also be used to detect the ripple effect of the changes in the software. The key difference between our work and Kung's approach is that we focus on the characteristics of the class and we identify the ripple effect of class characteristics that affect other classes. For example, we can identify attributes that become polymorphic as a result of the creation of an inheritance hierarchy.

Regression test selection techniques also track changes in software releases to identify test cases that can be reused to test the modified software. Rothermel et al. use a class control flow graph (CCFG) to represent the methods in a class[15]. To track the changes between two versions of a class the CCFGs for each class are constructed and each node in the graph is compared. The results of this comparison help the tester to identify the test cases to be rerun on the modified class. Our approach to track changes in a class is not as fine grain as the CCFG, however we do identify changes that the current version of the CCFG cannot detect. These changes include accessibility of features, polymorphic attributes or routine locals, and inherited characteristics. Our approach complements the CCFG in tracking changes.

\section{Concluding Remarks}

We have presented our taxonomy that enables a maintainer to catalog classes based on the properties of the class features, as well as the relationships with other classes. The properties of the class features include a summary of the types used, accessibility, shared class data, deferred features, polymorphism, dynamic binding, exception handling, and concurrency. The relationships include associations, dependencies and generalizations. Using our taxonomy tool we track the changes across various releases of a library application.

We are exploring the use of our taxonomy to generate a class integration test order for applications containing parameterized classes and concrete classes derived from an abstract class.

\section{References}

[1] Ken Arnold, James Gosling, and David Holmes. The Java(TM) Programming Language (3rd Edition). Addison-Wesley, 2000.

[2] K. Bennett and V. Rajlich. Software maintenance and evolution: A roadmap. In Proceedings of the conference on The future of Software engineering, pages 73-87, Limerick, Ireland, May 2000.

[3] P. Clarke and B. A. Malloy. A unified approach to implementation-based testing of classes. In Proceedings of ICIS' 01 , pages 226-234, October 3-5 2001.

[4] E. Gamma, R. Helm, R. Johnson, and J. Vlissides. Design Patterns: Elements of Reusable ObjectOriented Software. Addison-Wesley, 1995.

[5] R. Harrison, S. Counsell, and R. Nithi. An overview of object-oriented design metrics. STEP, 1997.

[6] M. J. Harrold, J. J. Jones, T. Li, D. Liang, A. Orso, M. Pennings, A. Sinha, and S. A. Spoon. Regression test selection for java software. In Proceedings of OOPSLA'01, pages 312-326, Tampa Bay, Florida, USA, June 2001.

[7] M. J. Harrold, J. D. McGregor, and K. J. Fitzpatrick. Incremental testing of object-oriented class structures. In Proceedings of ICSE, pages 68-80, Melbourne, Australia, March 1992.

[8] ISO/IEC JTC 1. International Standard: Programming Languages - $\mathrm{C}++$. Number 14882:1998(E) in ASC X3. ANSI, first edition, September 1998.

[9] D. Kung, J. Gao, P. Hsia, F. Wen, Y. Toyoshima, and C. Chen. Change impact identification in object oriented software maintenance. In Proceedings of ICSM, pages 202-211, British Columbia, Canada, September 1994.

[10] M. Lee, A. J. Offut, and R. T. Alexander. Algorithmic analysis of the impacts of changes to object-oriented software. In Proceedings of TOOLS 2000, pages 61-70, Santa Barbara, CA, USA, July 2000.

[11] S. Matzko, P. Clarke, T. H. Gibbs, B. A. Malloy, and J. F. Power. Reveal: A tool to reverse engineer class diagrams. In Proceedings of TOOLS, Sydney, Australia, Feb 2002.

[12] B. Meyer. Object-Oriented Software Construction. Prentice Hall PTR, 1997.

[13] J. F. Power and B. A. Malloy. Symbol table construction and name lookup in iso $\mathrm{C}++$. In Proceedings of TOOLS 2000, pages 57-68, Sydney, Australia, November 2000. 
[14] Simon Robinson, Burt Harvey, Craig McQueen, Christian Nagel, Morgan Skinner, Jay Glynn, Karli Watson, Ollie Cornes, and Jerod Moemeka. Professional C\# (Beta 2 Edition). Wrox Press Inc, 2001.

[15] G. Rothermel, M. J. Harrold, and J. Dedhia. Regression test selection for C++ software. Software Testing, Verification and Reliability, 10(2), June 2000.

[16] J. Rumbaugh, I. Jacobson, and G. Booch. The Unified Modeling Language Reference Manual. Addison Wesley Longman, Inc, 1999.

[17] B. G. Ryder and F. Tip. Change impact analysis for object oriented programs. In Proceedings of PASTE '01, pages 46-53, Snowbird, Utah, USA, June 2001.

[18] J. M. Vlissides and M. A. Linton. Iv tools. http://www.vectaport.com/ivtools/, March 2002.

[19] Whatis. Whatis.com target $\operatorname{search}^{T M}$. http://whatis.techtarget.com/, May 2002.

[20] N. Wilde and R. Huitt. Maintenance support for object-oriented programs. IEEE Transactions on Software Engineering, 18(12):1038-1044, December 1992. 